MS40-02

\section{The good, the bad and the ugly: small and macro molecular crystals in all their glory}

Elspeth Garman ${ }^{1}$

1. Department of Biochemistry, University of Oxford, Oxford, United Kingdom

email: elspeth.garman@bioch.ox.ac.uk

Both small and macromolecular crystals can be beautiful objects that are a joy to behold, both in real and reciprocal space. However, sometimes they can look optically perfect but give very disappointing (or no) diffraction or vice versa, and their behaviour in this regard is somewhat unpredictable [1]. Nonetheless, given a good, bad or ugly crystal, we can optimise the outcome of diffraction experiments by thinking about the conditions to which we subject the sample from the point of view of the crystal. This talk will expand upon the above theme, and use a cacophony of examples collected over 30 years of working in crystallography, ending with a particularly tricky one to illustrate the challenges that are sometimes faced in macromolecular structure determination by crystallography [2].

References:

[1]Owen, R.L.\&.Garman, E.F.. Acta Cryst (2005) D61, 130-140 [2] Abuhammad, A., Lowe, E.D., McDonough, M.A.,.Shaw Stewart, P.D., Kolek, S.A., Sim, E., \& Garman, E.F.. Acta Cryst. (2013) D69, 1433-1446

Keywords: crystals, diffraction, challenges

\section{MS40-03}

\section{Novel screening method for the crystallization of salts of organic cations}

Martin Babor ${ }^{1}$, Philipp Nievergel ${ }^{2}$, Jan Čejka ${ }^{1}$, Bernhard Spingler ${ }^{2}$

1. Department of Solid State Chemistry, University of Chemistry and Technology in Prague, Prague, Czech Republic

2. Department of Chemistry, University of Zurich, Zurich, Switzerland

email: Baborm@vscht.cz

Charged organic cations are an important part of chemical and especially pharmaceutical industry. Over $38 \%$ of all solid active pharmaceutical ingredients (APIs) are used in form of the cation with some suitable anion. Nowadays, there is a trend to use counterions other than the very popular chloride. [1] Different salts of the same API provide different physicochemical properties as solubility depending on $\mathrm{pH}$, stability, hygroscopicity and so on. Traditional salt screenings are time, material and labor work consuming. We present a new modification of the small-scale vapor diffusion crystallization technique of macromolecules with the purpose of anion screening for an organic cation. The basic ideas of our screening are an ion exchange and crystallization using vapor diffusion. This crystallization leads in most cases to high-quality single crystals measurable on an in-house X-ray diffractometer. The screening itself was performed with 7 structurally significantly different compounds, whose aqueous solubilities vary by a factor of almost 1000 . The screening was highly effective. At least one new salt was found for 6 out of 7 compounds. To summarize the screening, 18 new salts were found and structurally described. We are able to prepare about 550 crystallization experiments per hour. And the screening of 96 conditions consumes only between $26 \mu \mathrm{g}-22 \mathrm{mg}$ of a substance depending on their solubility. [2]

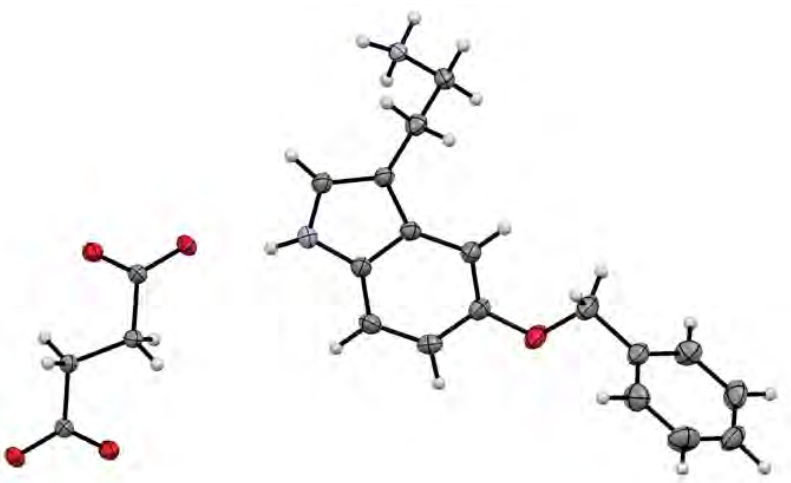

References:

[1] Paulekuhn, G. S., Dressman, J. B., \& Saal, C. (2007). Journal of Medicinal Chemistry, 50, 6665-6672.

[2] Nievergelt, P. P., Babor, M., Čejka, J., \& Spingler, B. (2018).

Chemical Science 9, 3716 - 3722.

Keywords: Salts screening, Pharmaceuticals, X-ray diffraction 